\title{
Swisscontact : Eine Selbstdarstellung
}

Swisscontact : un autoportrait

Robert Jenny

\section{OpenEdition}

\section{Journals}

Electronic version

URL: http://journals.openedition.org/sjep/951

DOI: 10.4000/sjep.951

ISSN: 1663-9677

\section{Publisher}

Institut de hautes études internationales et du développement

\section{Printed version}

Date of publication: 31 août 1982

Number of pages: 147-153

ISSN: 1660-5926

\section{Electronic reference}

Robert Jenny, « Swisscontact : Eine Selbstdarstellung », Schweizerisches Jahrbuch für

Entwicklungspolitik [Online], 2 | 1982, Online erschienen am: 24 Januar 2013, abgerufen am 08 September 2020. URL : http://journals.openedition.org/sjep/951 ; DOI : https://doi.org/10.4000/sjep 951

(c) The Graduate Institute 


\title{
SWISSCONTACT : EINE SELBSTDARSTELLUNG
}

\author{
Robert Jenny
}

Geschäftsleiter von Swisscontact

\section{SWISSCONTACT : UN AUTOPORTRAIT}

Résumé : Swisscontact a été créé en 1959 sur l'initiative du Professeur Jacques Freymond, sous la forme d'une Fondation suisse pour l'aide technique au développement. On espérait alors créer une classe moyenne de professionnels capables de prendre en main le développement de leur pays. Les subventions de l'industrie privée ont représenté au total, de 1959 à 1981, la somme de trente millions de francs. Depuis 1964, la Confédération participe également au financement; en 1981, sa part représentait environ $45 \%$ du budget.

Swisscontact est actif dans les domaines suivants : direction et organisation de cycles d'apprentissage technique, formation d'instructeurs, formation de techniciens, organisation de stages et de cours de recyclage, collaboration technique avec des coopératives, appui aux petites entreprises, résolution de problèmes techniques spécifiques par I'envoi d'experts du SEC (Senior Expert Corps). Swisscontact s'adresse principalement à un public déjà éduqué et qui veut poursuivre sa formation, donc à la classe moyenne, tout en admettant des priorités plus immédiates, confiées à d'autres organisations.

Les projets de formation dirigés par Swisscontact doivent être normalement confiés dans leur dernière phase à l'entière responsabilité des responsables locaux. Les partenaires sont en règle générale soit l'Etat, soit des organisations contrôlées au moins en partie par I'Etat. L'appui qu'offre l'économie privée à Swisscontact n'est pas lié à des intérêts commerciaux directs, quoique les avantages économiques qui peuvent découler des relations établies ne soient pas considérés comme néfastes. L'adaptation aux besoins des PVD amènera sans doute Swisscontact à devoir réviser ses critères de qualité. Le cas de I'Indonésie montre qu'il faut savoir trouver des compromis avec la quantité.

\section{Gründung und Entwicklung}

Swisscontact wurde 1959 auf Initiative von Professor Jacques Freymond, Genf, als Schweizerische Stiftung für technische Entwicklungshilfe gegründet. Das Ziel bestand darin, die schweizerische Privatwirtschaft über die kommer- 
ziellen Beziehungen hinaus zu einer Hilfe an die Länder Afrikas, Asiens und Lateinamerikas zu veranlassen. Man hoffte, mit der Ausbildung einer Mittelschicht von fähigen Berufsleuten, die in der Lage sind, eine eigenständige Entwicklung ihres Landes an die Hand zu nehmen, einen bescheidenen, jedoch realistischen Beitrag zur Verbesserung der wirtschaftlichen Verhältnisse der Dritten Welt zu leisten.

Die Beitragsleistungen der Privatwirtschaft betrugen von 1959 bis 1981 gesamthaft rund 30 Millionen Franken. Jährlich machen die freiwilligen Beiträge der Unternehmen nahezu 1,5 Million Franken aus. Seit 1964 gewährt der Bund finanzielle Beiträge und überträgt auch Projekte an Swisscontact in Regie. Der Anteil der Bundesmittel betrug 1981 etwa 45\% der gesamten Aufwendungen von Swisscontact in der Höhe von rund Fr. 7,8 Millionen. Diese über 17jährige Zusammenarbeit mit dem Bund hat sich als sehr wertvoll erwiesen und ist heute wesentlich für die Zukunft einer Organisation wie Swisscontact. Seit 1979 wird ein Projekt im Auftrag des indonesischen Erziehungsministeriums ausgeführt, das durch einen Kredit der IDA finanziert wird.

\section{Berufsbildung in Entwicklungsländern}

\section{Berufslehre}

Die Zielsetzung von Swisscontact war von Anfang an klar : mit dem Aufbau von Lehrwerkstätten, vor allem auf dem mechanischen Gebiet, wollte man begabten jungen Leuten in Asien, Lateinamerika und Afrika eine auf die Praxis ausgerichtete Ausbildung ermöglichen. Anfangs wurden in Asien und Lateinamerika vorwiegend Präzisionsmechanikerschulen erstellt. Später kamen andere Berufsrichtungen hinzu : Sanitärinstallateure, Schlosser, Automechaniker, Werkzeugmacher, Kältemonteure, Elektriker, Elektroniker usw. Diese Art von Berufsbildung ist vorwiegend ausgerichtet auf die Beschäftigung in der lokalen Industrie und dem Gewerbe. In ländlichen Gebieten und Ländern, die kaum industrialisiert sind, werden hingegen Unterhalts-, Landmaschinen- und Allroundmechaniker ausgebildet, die für die Wartung und Reparatur der an Ort und Stelle vorhandenen Geräte und Maschinen vor allem im landwirtschaftlichen Sektor benötigt werden. Lehrwerkstätten wurden oder werden in folgenden Ländern aufgebaut : Indien, Pakistan, Indone sien, Brasilien, Peru, Ecuador, Costa Rica, Sri Lanka, Tunesien und Tansania.

\section{Instruktorenausbildung}

Seit längerer Zeit wird in den Programmen von Swisscontact grosses Gewicht auf die Instruktorenausbildung gelegt, sei es im Rahmen der Vorbereitung einer geplanten Lehrwerkstatt oder aber unabhängig davon als Impuls für die technische Ausbildung eines Landes oder einer Region. Der Multiplikatoreffekt der eingesetzten Mittel steigt bei der Ausbildung der Ausbildner 
wesentlich im Vergleich zur Lehrlingsausbildung. Solche Kurse werden in Brasilien, Tansania und Indonesien durchgeführt.

\section{Technikerausbildung}

In Indonesien läuft ein gross angelegtes Programm zur Technikerausbildung. Man will damit eine Lücke zwischen dem meist sehr theoretisch geschulten Ingenieur und dem Handwerker ausfüllen.

\section{Kurzkurse und Weiterbildung}

Auf agromechanischem Gebiet werden Kurz- und Weiterbildungskurse direkt in den Dörfen angeboten. Mit mobilen Werkstätten, die für einige Wochen an einem Ort stationiert werden, finden Kurse für Unterhalt, Reparatur und Bedienung von Landmaschinen statt. In manchen Projekten werden auch Kurse für Erwachsenenweiterbildung durchgeführt, in einem Fall auch für administratives Personal. Kurzkurse finden in folgenden Ländern statt : Ecuador, Nicaragua, Guatemala, Costa Rica, Peru, Bangladesh, Lesotho.

\section{Zusammenarbeit mit Genossenschaften}

In Costa Rica, Peru und Ecuador arbeitet Swisscontact mit Genossenschaften zusammen und leistet einen Beitrag zur Verbesserung der organisatorischen Strukturen beim Einsatz der mechanischen Geräte. Dies beinhaltet die Mithilfe bei der Organisation von überbetrieblichen Maschinenstationen, Saatgutkontrolle, Anbauberatung, Düngung usw.

\section{Förderung von Kleinbetrieben}

Wir möchten Absolventen der von uns aufgebauten Lehrwerkstätten ermöglichen, erfolgreiche Kleinunternehmen zu betreiben. Durch Erteilung von Kleinkrediten, gekoppelt mit einer entsprechenden Betriebsberatung, sollen vorerst einmal Landmaschinenmechaniker unterstützt werden. Ein solches Programm läuft versuchsweise in Costa Rica an.

\section{Problemlösungen durch SEC-Experten}

Das Einsatzprogramm für pensionierte Fachleute, eine neue Initiative von Swisscontact, hat sowohl in der Schweiz wie in verschiedenen Entwicklungsländern Anklang gefunden. Allerdings ist es nicht ganz einfach, den richtigen Mann für die richtige Aufgabe zum richtigen Zeitpunkt zur Verfügung zu stellen. Im Gegensatz zu den bisherigen Programmen, geht es bei den SECEinsätzen nicht um den Aufbau von Projekten, sondern um die kurzfristige 
Lösung von klar begrenzten Problemen durch Spezialisten. SEC-Missionen dauern normalerweise nicht länger als drei Monate. Sie können irgendein fachliches Gebiet betreffen, da Swisscontact einzig die Vermittlung des Spezialisten übernimmt und kein eigenes Know-how benötigt. Es erfolgt keine Salärzahlung, sondern lediglich Spesenentschädigung. Die lokalen Kosten sind normalerweise durch den Auftraggeber, der staatlicher oder privater Natur sein kann, zu übernehmen.

\section{Die Philosophie von Swisscontact}

Es ist gar nicht einfach, das "Credo" einer vor allem pragmatisch orientierten Organisation in Thesen zu formulieren. Wir glauben nicht, dass sich allgemein gültige Rezepte für die Gesamtheit der Entwicklungsländer finden lassen, und wir finden es vor allem absurd, wenn Ideologien, die weitgehend in Europa entstanden sind, als Basis für die Formulierung einer Entwicklungspolitik herangezogen werden. Eine Entideologisierung im Verhältnis zur Dritten Welt und im Verhältnis zu unseren eigenen Anstrengungen im Rahmen der Entwicklungszusammenarbeit erscheint uns vordringlich. Dazu gehört vor allem auch eine Entspannung im Verhältnis zwischen den Exponenten der Entwicklungszusammenarbeit und denjenigen der Wirtschaft, die sich gerade im vergangenen Jahr in der Schweiz in eine vollkommen unfruchtbare Konfrontation hineingesteigert haben.

Die Leitlinien für die Tätigkeit von Swisscontact lassen sich grosso modo folgendermassen formulieren :

\section{Prioritäten}

Wir anerkennen durchaus die Vordringlichkeit der Entwicklung der Landwirtschaft und der Hilfe an die ärmsten Entwicklungsländer. Andererseits kann kein Land eine gewisse Entwicklungsschwelle überschreiten, ohne auch eine angepasste Industrialisierung anzutreben. Dass dies auch in Entwicklungsländer erkannt wird, wo eine sogenannte eitenständige Entwicklungspolitik verfolgt wird, zeigen mannigfache Beispiele. Es erschiene uns ferner unsinnig, aus dogmatischen Ueberlegungen die Bedürfnisse der sogenannten Schwellenländer zu vergessen und Gefahr zu laufen, dass diese wieder auf einen tieferen Entwicklungsstand zurücksinken. Es ist deshalb die bewusste Absicht von Swisscontact, diese vielleicht zweit- oder drittrangigen Prioritäten, die aber immer noch Prioritäten sind, als Zielrichtung anzugehen. Dies gilt auch für die Bevölkerungsschichten, die unmittelbar von unseren Projekten profitieren. Es sind dies kaum die Aermsten der Armen, sondern es sind vorwiegend junge Leute mit einer relativ guten Schulbildung, denen überhaupt eine erfolgreiche Teilnahme an einem technischen Unterricht möglich ist. Es ist unser Ziel, einen beruflichen Mittelstand zu fördern, in der Hoffnung damit auch einen Beitrag zu Stärkung eines sozialen Mittelstandes zu leisten. Dabei versuchen wir allerdings mit unseren unformellen Ausbildungsprogrammen, auch andere, benach- 
teiligtere Schichten zu erreichen, vor allem durch Kurzkurse in ländlichen Gebieten.

\section{Methode}

Unser Spezialgebiet ist die Ausbildung, vor allem auf technischem Gebiet. Bei aller Problematik des Einsatzes von sogenannten Experten aus Industrieländern in der Dritten Welt ist eine wirksame Uebertragung von solidem, technischem Können nur durch entsprechende Fachleute möglich. Unsere Projekte werden deshalb ausschliesslich durch Personal an Ort und Stelle im Entwicklungsland realisiert, wobei wir allerdings von Anfang an eine systematische Uebergabeplanung vorsehen. Normalerweise wird ein Ausbildungsprojekt von unseren Mitarbeitern in den ersten Jahren selbst geführt, wobei sukzessive die Verantwortung einem lokalen Counterpart übertragen wird. Anschliessend erfolgt eine Uebergabe der Gesamtverantwortung, wobei die Betreuung durch unsere Mitarbeiter noch kurze Zeit weiterläuft. Nach unserem Weggang schliesst sich eine sog. Follow-up Phase mit Unterstützung aus Distanz an. Unsere bisherigen Erfahrungen mit den übergebenen Projekten sind trotz einigen Enttäuschungen im allgemeinen positiv.

\section{Partnerauswahl}

Wir arbeiten in erster Linie mit staatlichen oder halbstaatlichen Partnern zusammen, obschon wir die Problematik dieser Wahl durchaus kennen. Das Berufsbildungswesen ist aber in den meisten Ländern zu einem grossen Teil vom Staat kontrolliert, weshalb wir uns durch dieses Vorgehen eine bessere Kontinuität und eine breitere Ausstrahlung versprechen, wenn auch unmittelbar eine geringere Effizienz als bei der Zusammenarbeit mit privaten Institutionen resultiert. Ein Hauptproblem, mit dem wir konfrontiert werden, liegt in der ungenügenden Bezahlung der lokalen Ausbildner durch die staatlichen Stellen, weshalb diese ständig die Tendenz haben, in die Privatwirtschaft abzuwandern. Bei der Partnerwahl richten wir uns übrigens nach den speziellen Verhältnissen im betreffenden Land und gehen sehr pragmatisch vor. So arbeiten wir mit staatlichen Berufsbildungsorganisationen, verschiedenen Ministerien aber auch mit einer technischen Universität, Forschungsinstitutionen und im Rahmen der agromechanischen Projekte mit Kooperativen zusammen.

\section{Verhältnis zur Privatwirtschaft}

Swisscontact ist eine neutrale Organisation, die aber aus Kreisen der Privatwirtschaft hervorgegangen ist und nach wie vor finanzielle Beiträge der Privatwirtschaft erhält, während keine öffentlichen Sammlungen durchgeführt werden. Wir glauben, dass vor allem die schweizerische Industrie wesentliche Impulse und Know-how für die Entwicklung in der Dritten Welt vermitteln kann und dies auch auf kommerzieller Ebene durch Investitionen und Technologietransfer tut. Die Unterstützung von Swisscontact durch die Privat- . 
wirtschaft hat aber mit direkten kommerziellen Interessen nichts zu tun, es sei denn, dass dadurch der Goodwill gegenüber der Schweiz und ihrer Wirtschaft gefördert wird. Unseres Erachtens tut es aber den Anstrengungen eines Industrielandes auf dem Gebiet der Entwicklungszusammenarbeit keinerlei Abbruch, wenn als Nebenprodukt auch gewisse Vorteile für das Geberland resultieren - immer unter der Voraussetzung, dass primär die Interessen des Entwicklungslandes eindeutig im Vordergrund stehen. Auf lange Frist wird eine fruchtbare Zusammenarbeit zwischen Industrieländern und Entwicklungsländern nur aufgrund der Erkenntnis gemeinsamer Interessen tragfähig sein. Die reine und selbstlose Solidarität zwischen Staaten gibt es ganz einfach nicht.

Von radikalen Abkoppelungstheorien halten wir nicht viel. Die Aktivität der Privatwirtschaft in der Dritten Welt hat nebst vielen positiven sicher auch negative Effekte. Wenn eine bessere Koordination zwischen staatlichen und wirtschaftlichen Kräften vorhanden wäre, liesse sich vielleicht das Potential der schweizerischen Industrie noch besser für die prioritären Entwicklungsbedürfnisse einsetzen. Massive staatliche Interventionen und Restriktionen von seiten der Industrieländer wirken aber in erster Linie lähmend und dienen kaum den Interessen der Entwicklungsländer. Das Tempo und die Art der Industrialisierung müssen weitgehend durch die Entwicklungsländer selbst gesteuert werden, was bei den meist undemokratischen und oft chaotischen politischen Verhältnissen zugegebenermassen leider sehr oft nicht im Sinne der wirklichen Interessen des betreffenden Landes erfolgt.

\section{Abgrenzung gegen andere Privatorganisationen}

In unserer Projektarbeit unterscheiden wir uns, abgesehen von der Spezialisierung, wohl kaum wesentlich von verschiedenen anderen Organisationen, die auch Fachleute in Entwicklungsländern einsetzen. Hingegen ist unsere Informationstätigkeit in der Schweiz relativ limitiert, da wir keine öffentlichen Sammlungen durchführen, sondern gezielt in der Privatwirtschaft, bei Gemeinden und Kantonen Finanzbeschaffungsaktionen unternehmen. In politische Auseinandersetzungen schalten wir uns nicht ein, wenn es nicht um direkte Anliegen der technischen Entwicklungszusammenarbeit geht, wie z.B. bei der Vernehmlassung für das Gesetz über die Entwicklungszusammenarbeit.

Mit den anderen grösseren Privatorganisationen haben wir regelmässige Kontakte, heute vor allem auch im Rahmen der Intercooperation, zu deren Gründungsmitgliedern wir gehören. Selbstverständlich sind wir in ständigem Kontakt mit der DEH, die rund die Hälfte unserer Aktivitäten finanziert und uns auch immer wieder Regieprojekte überträgt. 


\section{Zukunftsperspektiven}

Unsere traditionelle Aufgabe im Rahmen der Berufsbildung wird noch auf Jahre hinaus Schwerpunkt unserer Tätigkeit bleiben. Immer wieder müssen wir aber überprüfen, ob die Stossrichtung noch stimmt, vor allem ob wir den Bddürfnissen des betreffenden Landes gerecht werden und nicht Leute ausbilden, die vielleicht gar nicht in erster Linie benötigt werden. So sind wir ja heute zu einem guten Teil auf einfachere Berufsgattungen, vor allem im Unterhaltsbereich, und zu informeller Kurzausbildung übergegangen. Daneben fordern uns aber Länder wie Indonesien, die gewaltige Ausbildungsbedürfnisse haben, auch auf anderen Ebenen, die zum Teil etwas von unserer Praxisorientierung wegführen. Die jährlich etwa tausend sechs hundert Techniker, die in Zukunft in den von uns zu betreuenden Schulen ausgebildet werden, sollen aber die Zwischenstufe zwischen Praktiker und Ingenieur ausfüllen, was ebenfalls einem wichtigem Bedürfnis entspricht. Ob wir in Grossprojekten, wie sie sich nun in Indonesien anbahnen, unsere schweizerischen Masstäbe noch halten können, ist zu bezweifeln. Bis anhin haben wir immer Qualität vor Quantität gesetzt, müssen aber erkennen, dass ein Land wie Indonesien Kompromisse einzugehen hat. Diese zu akzeptieren ist oft schwierig für einen seriösen schweizerischen Fachmann.

Eine in der Entwicklungszusammenarbeit tätige Organisation muss sich aber auch selbst entwickeln und darf nicht in der Routine stehenbleiben. Nebst den bewährten Programmen führen wir deshalb immer wieder Versuchprojekte durch, in einer Grössenordnung, die auch einen Fehlschlag verschmerzen lässt. In diese Kategorie dürften wir wohl die Gewerbeförderung, die Ausdehnung der Zusammenarbeit mit den Kooperativen in Lateinamerika auf die Anbauberatung, den Versuch, Ausbildung durch wirtschaftliche Produktion zu ergänzen, und schliesslich dac Senior Expert Corps einreihen.

Last but not least wäre noch etwas über die Aussichten in bezug auf den Ausbau der finanziellen Basis zu sagen. Die Chancen für eine wesentliche Erhöhung der Beiträge der Privatwirtschaft stehen in diesen wirtschaftliche unsicheren Zeiten nicht sehr gut. Andererseits können wir unsere Erfahrung auf unserem Spezialgebiet dem Bund und anderen Auftraggebern, wie Entwicklungsbanken, anbieten. Zur Zeit beschäftigen wir im Ausland über fünfzig Mitarbeiter. Diese Zahl wird sich im nächsten Jahr weiter erhöhen, da uns der Bund ein grösseres Projekt im Rahmen der technischen Lehrerausbildung in Indonesien überträgt. Eine Fortsetzung unserer Tätigkeit für die indonesische Regierung und die IDA steht ebenfalls in Aussicht. Damit dürften unsere Aktivitäten in nächster Zeit noch weiter anwachsen. Dies soll jedoch mit Mass geschehen, da wir weiterhin gemäss der Devise "small is beatiful" nicht auf die Vorteile und die Flexibilität einer kleinen Organisation verzichten möchten. 